\section{La belleza como valor emocional en el espacio cultural}

Esther Giani ${ }^{(1)}$

\begin{abstract}
Resumen: La belleza salvará el mundo. Pocas son las frases que han sido utilizadas tanto como esta. La belleza se va manifestando de forma cada vez más impetuosa. Nos referimos a la exigencia de acceder a la belleza como "alto conocimiento" en los espacios culturales. Ptolomeo representó la producción artística y cultural situándola en un preciso lugar: un edificio de culto, reservado a pocos elegidos. Desde los años noventa, el Museo se convierte en un terreno de confrontación internacional para comprobar las políticas culturales de varios países. Se abre así un ámbito de intereses financieros, estrechamente relacionados con la producción de bienes inmateriales; lo público y lo privado figuran en algunas ocasiones como antagonistas y en otras como aliados, incrementando el establecimiento de museos privados que abren al público sus propias colecciones. La desmembración del saber en competencias cada vez más especializadas ha modificado la propia representación del conocimiento, y los museos lo han aprovechado recolectando memorias históricas, sociales y de costumbre cada vez más fragmentadas. Para definir una posible idea futura de Museo, dos aspectos necesitan ser esclarecidos: primero qué será imprescindible recopilar, ordenar y transmitir, y segundo, y más importante, cómo hacerlo. ¿Qué es lo que se puede hacer para atar fragmentos del tiempo presente, aquellos de una mentalidad contemporánea, los de una compartida sensibilidad estética? Este asunto involucra cuestiones éticas profundas, abriendo el debate sobre los sistemas funcionales y económicos que se traduce en un llamamiento para responsabilizarnos de cara al futuro, y que desafortunadamente se elude a menudo debido a las lógicas económicas dominantes.
\end{abstract}

Palabras clave: Belleza - Espacios Culturales - Paradigma Material - Arqueología Del Presente.

[Resúmenes en inglés y portugués en las páginas 82-83]

(1) Profesora de composición arquitectónica y urbana desde 2019, fue investigadora e investigadora. Tiene un doctorado en Composición Arquitectónica con una tesis sobre las Escuelas Nacionales de Arte de La Habana y obtiene una maestría del Instituto Berlage con una investigación sobre las posibilidades de la arquitectura para reparar barrios degradados con el sistema urbano. En 1998 se graduó en arquitectura en Venecia, con una tesis sobre el paisaje industrial de Marghera. Ha pasado largos períodos de estudio e investigación en el extranjero y ha tenido experiencias de diseño en talleres internacionales desde 1992. Desde el año 2000 ha estado enseñando en Iuav y ha estado desarrollando su investigación 
dentro del grupo "áreas en desuso y paisajes industriales" iniciado por Giancarlo Carnevale con quien colabora. Para Iuav coordinó el WAVe (2007-12); para el Departamento de Arquitectura, Conservación de la Construcción, fue miembro del consejo, la comisión conjunta y esa pasantía (2015-18), también coordinó los talleres "Una semana con ..." (2018-19). A partir de 2019 es responsable de los estudiantes entrantes Erasmus. Coordina trabajos de investigación y profesores visitantes.

Es muy activa en la difusión de los resultados alcanzados, tanto didácticos como de investigación. Además de numerosos números de "Giornale Iuav", en 2019 es la autora del primer compendio sobre Porto Marghera ( Porto Marghera Atlas ) con I. Peron; y días de estudio relacionados ( Sconfinamenti. Obras de Le Corbusier en el Iuav de Venecia ) En 2014 con G. Carnevale sobre la composición arquitectónica escribió Il Nuovo che arretra / The Reatreat of the New. Participó con los proyectos en la Bienal de Venecia (2004 y 2010), en la Trienal de Milán (2006). Asiste regularmente a conferencias, concursos de diseño, investigación y exposiciones internacionales.

\section{Introducción}

La belleza salvará el mundo ${ }^{1}$.

(Dostoevskij)

Pocas son las frases que han sido utilizadas tanto como esta. En la obra El Idiota de Fiódor Dostoievski, la pronuncia el príncipe Miškin, aunque el contexto es totalmente diferente respecto a lo que se suele considerar hoy en día. La riqueza semántica de la alegación, junto con su inclusividad sintética y concluyente, hacen que se pueda considerar un passepartout ${ }^{2}$ irresistible. La idea de Belleza Salvífica representa una exigencia omnipresente, que en las últimas décadas se va manifestando de forma cada vez más impetuosa. Nos referimos a la exigencia de acceder a la belleza como "alto conocimiento", conocimientos que solían ser reservados a una elite estricta. Ptolomeo ${ }^{3}$ tuvo ya la idea de representar la producción artística y cultural de forma ordenada, agrupando todo en un solo lugar; sin embargo, este lugar estaba pensado como lugar de culto y por lo tanto reservado solo a unas pocas personas elegidas.

Sin revisar la evolución de la institución Museo de los últimos años, queremos destacar que solo a partir de la década de los noventa se convirtió en un fenómeno cultural de masa y, por lo tanto, en un business. El desarrollo del Museo como institución, a nivel mundial, corresponde a la representación de la propia historia, de la propia cultura, de las tradiciones, de las raíces. Se convierte así en un terreno de confrontación internacional para comprobar las políticas culturales de varios países, como una lucha (simbólica, por supuesto) entre Poderes Nacionales. La financiación pública se hace cada vez más importante como consecuencia del aumento de la demanda; el patrón del turismo se ha modificado, con la consecuente introducción de nuevas estrategias de gestión y satisfacción (accesos limita- 
dos, reservas, oferta particular diversificada, exposiciones temporales, nuevas gestiones administrativas, patrocinadores, fundaciones ad hoc). Se crea así un conjunto de intereses financieros estrechamente relacionados con la producción de bienes inmateriales. Lo público y lo privado, según la ocasión, pasan de ser antagonistas a ser aliados (sobre todo en el mercado de las artes visuales contemporáneas) y viceversa, llegando al punto donde existe un incremento de museos privados que exponen al público sus propias colecciones ${ }^{4}$. Un inciso: actualmente, está teniendo lugar una gran controversia respecto a la definición de Museo. En el 2007, la ICOM ha definido Museo como:

Institución permanente sin fines de lucro, al servicio de la sociedad y de su desarrollo, abierta al público y que se hace cargo de investigar testimonios materiales e inmateriales sobre el ser humano y su entorno, su recopilación, conservación y concretamente su exposición, con un fin didáctico y de entretenimiento ${ }^{5}$.

En la actualidad, hay un nuevo jurado que se está encargando de renovar dicha definición. Esto significa que nos estamos enfrentando a una evolución tan rápida de los paradigmas tradicionales críticos y técnicos que se hace cada vez más necesaria una profunda revisión de la idea de Museo, y como consecuencia de su misma definición. Sin embargo, estas definiciones pueden ser muy interesantes ya que son potencialmente ricas de oportunidades a muchos niveles, para los países que tienen más geografía que historia. Esto es claramente perceptible visitando las Bienales de Arte de Venecia del último lustro.

De hecho, la diferenciación y fragmentación del conocimiento para competencias cada vez más especializadas han dado lugar a cambios que afectan hasta a la misma representación del conocimiento. Los museos lo han aprovechado para convertirse en lugares donde se celebran y se recolectan memorias de segmentos históricos, sociales y de costumbre que resultan ser cada vez más fragmentadas. Hoy en día, existen museos que recopilan o objetos de cada tipo: museos de muñecas, de balanzas, etc. Además, el desarrollo de la cultura material $^{6}$ en los últimos tiempos, determina la creación tanto de nuevas colecciones como de una extensa y difundida idea de ambiente museístico?

El hecho de que al edificio Museo se le haya atribuido un rol de comunicación autónoma y de espectáculo, representa una fuente de confusión adicional. Resultaría excesivo centrarse sobre la tendencia, ya predominante, a elaborar expresiones arquitectónicas ajetreadas, teatrales, como si se estuviera delante de máquinas de fiesta de temporada (aunque la arquitectura se quedará como testigo por generaciones...). Generalmente, las Obras Públicas y en concreto los Museos, representan cada vez más una oportunidad perfecta para el exhibicionismo arquitectónico puro. Claramente, no representa la única alternativa viable, aunque esto es lo que se ha impuesto y que resulta firme en el imaginario contemporáneo. Además, es necesario destacar la propensión a considerar como producto de la cultura contemporánea lo que procede de ámbitos tanto artísticos como científicos, siguiendo el mismo patrón de organización en repertorios y ábacos. Habitualmente se han utilizado categorías, clasificaciones e inventarios, aunque en la actualidad adoptan el significado de categoría estética y paradigma comunicativo. Con la complicidad del universo digital, nos encontramos rodeados de todo tipo de sistemas ordenadores, variados y fantasiosos; esta 
nueva realidad está influenciando los mecanismos de comunicación y, consecuentemente, nuestra manera de vivir y de interactuar en todos los niveles.

Recordemos que, para Ortega y Gasset, las "modas" no son algo que puedan ser tomadas a la ligera ${ }^{8}$, puesto que definen actitudes difusas, mentalidades ${ }^{9}$ presentes y fuerzas históricas que dejarán rastro; $y$, por lo tanto, tienen que ser tomadas en cuenta.

Lo que nos interesa definir es un posible escenario futuro para la Idea de Museo, enfocándonos en dos asuntos principales -como ya ha escrito antes: en primer lugar, hay que establecer lo que se necesitará recopilar, testificar y transmitir; y, en segundo lugar, se necesitará determinar la manera para llevar todo a cabo. Seguramente, no se hará nada que implique el embalsamamiento de los lugares que pertenecen a su propio tiempo, quitando lo que define la vida y el uso de sus habitantes. Hasta ahora, otros campos se han hecho cargo de transmitir estos conceptos, como definición de lugares urbanos basada en los cambios que el paso del tiempo ha dejado: es el caso, por ejemplo, de literatura, fotografía, archivos de imágenes urbanas, cine y todas las artes figurativas que han intentado captar el espíritu del tiempo, el espíritu de segmentos históricos concretos.

Antes de elaborar un posible escenario para un novedoso concepto de Museo, llamamos una vez más la atención sobre el estudio que Georges Perec quería emprender. Tentativa de agotamiento de un lugar parisino ${ }^{10}$ es el texto que indica el principio de lo que se definió como "experimento etnoantropológico": se trataba de describir minuciosamente doce lugares de Paris, cada año durante doce años, con la intención de registrar "el envejecimiento de los lugares, el envejecimiento de la escritura, el envejecimiento de los recuerdos..."11. Aunque Perec no consiguió terminar su proyecto, no pierde fuerza la influencia que esta idea ejerce y nos transmite. ¿Qué se puede hacer para que puedan asentarse las piezas del presente que hasta ahora han sido consideradas irrelevantes (ordinarias e informales), que sin embargo son documentos valiosos e imprescindibles para transmitir tanto la cultura material ${ }^{12}$ como la mentalidad de su propio tiempo, con su proprio sentido del decoro y su sensibilidad estética? ¿Cómo se puede prevenir el embalsamamiento kitsch que se traduce en una folclórica representación escénica?

La discusión no puede prescindir de considerar aspectos éticos y profundos, lo que implica abrir el debate sobre los mecanismos funcionales y económicos, y nos invita a considerar la responsabilidad que tenemos hacia el futuro, y que a menudo se elude por sucumbir a las lógicas económicas dominantes. La arqueología del presente también se refiere a la arquitectura; sin embargo, no ha sido aun desarrollado un verdadero debate sobre este tema ${ }^{13}$.

Nos gustaría centrarnos en las oportunidades que las tecnologías contemporáneas están ofreciendo para conservar lo que queremos transmitir en el futuro sobre nuestro mundo y nuestra forma de vivirlo y habitarlo. ¿Qué saben los jóvenes de hoy sobre lo que era Londres en los tiempos de Mary Quant y de los Beatles? Se entiende perfectamente la imposibilidad de reproducir la misma atmósfera que se queda ahora dispersa y pálida, presente solo en algún que otro fotograma de viejas películas. Lo cierto es que esos lugares, colores, sonidos y el ambiente en su totalidad, se ha perdido para siempre. Sin embargo, hoy tenemos a nuestro alcance los medios tecnológicos para que la realidad pueda ser registrada con una gran fidelidad. Existen aplicaciones que permiten la visita virtual de todos los museos ${ }^{14}$. 
Rem Koolhaas, en el 2012 en Venecia ${ }^{15}$, respaldó la necesidad de preservar para las generaciones venideras, no solo lo que se presenta como monumento o reliquia, si no también todo lo que pueda representar la mentalidad difusa y el espíritu del tiempo ${ }^{16}$. Actualmente, la realidad aumentada es una herramienta a nuestro alcance y podría recuperar el innato interés en el proyecto de Perec. O sea crear un archivo digital cuyo tamaño sea tal que pueda contener todo lo que pueda definir un lugar urbano, como detalles materiales que ocupan un espacio físico en su entorno, incluyendo aquellos inmateriales como sus ruidos, olores y flujos de todo tipo que lo atraviesan. Cualquier objeto, incluso el más modesto, si se puede transmitir en el tiempo, adquiere valor documental ${ }^{17}$. Dicho valor aumenta su interés en el futuro. Por ejemplo, se puede comparar el valor que tiene una vasija cerámica diaguita precolombina con las piezas de vajilla traídas por los inmigrantes europeos a la Argentina.

Lo cotidiano es un valor que desde siempre se subestima: solamente los artistas han conseguido intuir su importancia, anticipando a científicos e historiadores. Al día de hoy se podría hacer más, yendo más allá del extraordinario trabajo testimonial que, de forma casi nunca institucional (como tiene que ser), los artistas continúan su desempeño, indagando las fuerzas históricas que atraviesan las conciencias, y que dejan en las ciudades rastros que resultan a veces impactantes y otras veces, extremadamente sutiles. Aquel espíritu del tiempo tan escurridizo, puede quedarse enredado solo parcialmente en los testimonios artísticos que sobreviven. Hoy en día, en efecto, somos más conscientes de la influencia que tiene "el estilo de vida" y, por lo tanto, de lo importante que es observarlo con atención (Ortega y Gasset). Los signos que se van inscribiendo de manera atemporal en los espacios que habitamos, son pistas y síntomas que anuncian los cambios en proceso, de tendencias y de fuerzas históricas que se van agregando: el espíritu de tiempo, el Ángel de la Historia que mueve suavemente sus piezas ${ }^{18}$.

Hay otro tema que merece la pena debatir: ¿es necesario transmitir el recuerdo de estos aspectos cotidianos menores y de las atmósferas que, en un momento concreto, definen la escena urbana? A esa pregunta se suele contestar afirmativamente. Es cierto que los documentos de la cotidianidad tienen mucho valor y resultan sumamente útiles a la hora de entender la cultura, el gusto, la mentalidad de una época y el sentido de los mismos monumentos y de las obras maestras consagradas. El estado del arte ya considera como documentos preciados, que bajo ningún concepto se puedan perder, tanto la carta de un soldado de la primera guerra mundial como la lista de la compra de la familia de la tía de San Martin y también los grafitis en un prostíbulo de Pompeya.

Lo que todavía falta es una investigación concluyente sobre las estrategias y los criterios para seleccionar, con continuidad, muestras de ciudades, de espacios urbanos y no en diferentes segmentos temporales, entregando a nosotros mismos, y sobre todo al futuro, los repertorios y los documentos que capturen el Zeitgeist $t^{19}$ que tiene formas, colores, volúmenes y esencias. Y esta esencia se puede hoy sintetizar, claramente solo en parte y con cierto grado de aproximación, con técnicas innovadoras que en el pasado no estaban a nuestro alcance. 


\section{Conclusión}

Creemos firmemente que es necesario apoyar un estudio que defina los criterios para poder seleccionar los fragmentos del presente y entregarlos a la Memoria. No pensamos en una representación que cristaliza los eventos (la referencia está en la crítica de la revista "Les Annales" a la historia événementiel - de los eventos): sin embargo, es más arduo individuar fragmentos "infraordinarios" que, por su modesta cotidianidad, se perderán para siempre. Creemos que esta es una responsabilidad histórica ineludible, sobre todo en una época de cambios descontrolados cuyas consecuencias resultan ser, de momento, impredecibles.

Deberíamos construir este escenario de estudios y realizaciones sintéticas para que se pueda alcanzar un entendimiento más completo de La Belleza Salvará el Mundo, refiriéndonos concretamente a la realización de un museo de la memoria, quizás desmaterializado (pero por supuesto con centros físicos de investigación, producción y hasta de consumo) que nos permita "salvar" los valores estéticos al igual que las atmósferas, entendidas como todas aquellas condiciones mentales que por su propia naturaleza resultan indescriptibles, evasivas y pasajeras, y que sin embargo representan la identidad más profunda de los momentos históricos que los generan.

\section{Notas}

1. Dostoevskij, F. (1869) - El Idiota. En realidad, Dostoevskij se refería a una belleza ética y no estética; la palabra rusa Mir (la construcción original de la frase es Mir spasër krasotà) podría significar también Paz, pero su interpretación "romántica" ha sido irresistible por el encanto evocativo que el concepto de Bello siempre transmite. Un ejemplo: el ensayo de Cvetan Todorov (2006) - Les aventuriers de l'absolu, ha sido traducido Los aventureros del absoluto, y La belleza salvará al mundo (Galaxia Gutenberg, 2007).

2. Passpartout es una palabra francesa para definir algo que funciona para todo: una clave de acceso o una clave maestra.

3. Para Ptolomeo (approx. 100 d.C.), el Museion erigido en Alejandría era el lugar de las Musas, una institución cultural abierta y cuya misión era la búsqueda y la difusión de la Belleza. Únicamente a partir del siglo XX, la palabra Museo se refiere a colecciones de objetos con valor estético o documentario.

4. Ha quedado constancia que en el pasado también se han alojado colecciones en museos privados (por ejemplo, los Jacquemart André y Nelly en Paris, los Guggenheim, el Gulbenkian en Lisboa para citar algunos ejemplos). La novedad está en el enlace más "activo" entre el acto de coleccionar y el mercado: las colecciones no se exponen al público solo por vanidad y prestigio personal, sino para que suba su valor económico. Actualmente, se observa un cambio convulso en las relaciones entre la critica "militante", el coleccionar, los museos y las galerías de arte.

5. La International Council of Museum (ICOM) es la principal organización internacional que representa tanto a los museos como al personal profesional. En su página web se 
declara que "la organización atiende a la comunidad museística en su misión de cuidar, conservar y compartir el patrimonio cultural presente y futuro, material e inmaterial". En el mundo globalizado en el que vivimos, el ICOM se presenta como la única institución "habilitada" para hablar de Museos...

6. "El concepto de cultura material apareció en las Ciencias Humanas, especialmente en la Historia, posteriormente a la institución de Antropología y Arqueología, y también a la influencia ejercida por el materialismo histórico. (...) El estudio de la cultura material (...) enfoca los hechos repetidos, y no en los eventos; no trata las superestructuras, sino las infraestructuras. (...) La cultura material aspira finalmente a crear un enlace con la imaginación del hombre y su creatividad, considerando como suyas tres componentes fundamentales: el espacio, el tiempo y la capacidad social de los objetos". Bucaille, R. y Pesez, J. M. (1993), p. 271-305. [E.Z.]

7. Véase: Giani, E. (2015) - Landscapes of Repetance and of Compensation, Procedeengs; Carnevale, G., Giani, E. (2015) - The Urban Scene; Carnevale, G. (2015) - Interstizi; Carnevale, G., Giani, E. (2014) - Occasioni di Rierca. Il Nuovo che Arretra // Research Opportunities. The retreat of the new; Carnevale, G. (2012) - Paradigmi Alieni. Se vean tambien de Carnevale, G. Architettura Grottesca, Una Non Evitabile Opportunità (1999), Grottesco (1995), Deformazioni ai Margini (1993) y Il Grottesco Prossimo Venturo (1989).

8. En el ensayo Estudios sobre el amor (1926), J. Ortega y Gasset dice: "Afirmo que hasta las cosas que se consideran más serias se desarrollan y se mutan siguiendo los mecanismos esenciales de la moda, que por lo tanto se convierte en ley fundamental de la realidad, evidenciando que las cosas sigan este curso, porqué así tiene que ser]". Añade poco después "[hay también que decir que las modas, en contextos de aparentemente menor relevancia -ropa, hábitos sociales etc.- poseen un significado mucho más profundo y serio de lo que, con frivolidad, se le suele atribuir". In ID (1993/2006) - La scelta in amore, p. 62. [E.Z.]

9. Lo que se presenta en la obra Storia delle mentalità, es un ámbito de estudios que desarrolla tanto los fundamentos teóricos como la práctica de la investigación en el contexto de la revolución historiográfica del siglo XX, bajo la influencia de la escuela École des Annales: (fundada por M. Bloch y L. Febvre en 1929). La tercera generación de los “Annales" (J. Le Goff y P. Nora, 1970) se encargó de enfocar los estudios y las teorías para crear una nueva historia, recogiendo tanto los aspectos culturales como mentalidades y representaciones. 10. “Octubre 1974. Durante tres días seguidos, George Perec se sienta en las mesas de los cafés o en los bancos en Place Saint-Suplice, 6o arrondissement, Paris, y observa la plaza en diferentes momentos del día. Toma cuidadosamente notas sobre todo lo que ve: personas, coches, autobuses, animales, nubes, cosas que parecen ser insignificantes, y sin embargo dan vida a la gran ciudad. Los innumerables e imperceptibles cambios de tiempo, luz, hojas, sombras y colores, se captan desde una perspectiva única y vibrante, que nos devuelve una obra maestra, un clásico, que se ha vuelto a publicar a los treinta años de la muerte de su autor". G. Perec (1975), contraportada de la traducción italiana del 2011 - Tentativo di esaurimento di un luogo parigino. [E.Z.]

11. En 1969 Perec empezó a trabajar sobre un proyecto muy ambicioso de escritura dual: describir 12 lugares de Paris (calles, plazas, passages, cruces), que estaban vinculados a la historia personal del escritor. Cada uno de estos lugares tenía que ser descrito in situ una vez al año, de la forma más neutral posible. Más adelante, en otro momento del mismo 
año, tenían que sacarse a la luz las memorias vinculadas al lugar. La evocación de dichos recuerdos tenía que llevarse a cabo lejos del lugar al que se referían. Un algoritmo matemático (bicuadrado latino de orden 12, creado especialmente para Perec por el matemático indio Chakravarti) disponía los 12 lugares en orden, al fin de evitar repeticiones y superposiciones; finalmente, los textos terminados se sellaban dentro de un sobre. En estos sobres también se podían incluir fotos o pruebas que comprobaran la presencia del escritor en dichos lugares (billetes del metro, recibos, tickets del cine, folletos). Perec tenía previsto el trabajo en 12 años con 288 sobres sellados. En 1973, cuarto año de trabajo, Perec no dice gran cosa sobre lo que le pasará al proyecto Lieux [Lugares] cuando, en enero 1982, sean abiertos los sobres: "Solo en aquel momento sabe si ha valido la pena. De hecho, no espero nada más que el rastro de un envejecimiento triple: el de los lugares, el de mis recuerdos y el de mi escritura". Lieux sigue inconcluso [E.Z.]

12. Con respecto a la cultura material, citamos algunos de los trabajos de Giancarlo Carnevale: la voz Cultura Material en el Dizionario critico illustrato delle voci piú utili dell'architetto moderno, de L. Semerani (1993); el volumen A regola d'arte (2006), el ensayo "Second life" in Carnevale, G. y Giani, E. Occasioni di ricerca overo il nuvo che arretra, pp. 65-87.

13. Véanse otros trabajos de la autora: For an operante archeology of the present. Case studies: vernacular Venice and Berisso y Ensalada (La Plata, Argentina) con L. Valera, presentado en HERITAGE 2016, y Travel in time. Is the informal a heritage to be preserved? Por REHAB 2015.

14. Si el dramaturgo francés Antonin Artaud ha sido el primero en definir el teatro como "réalité virtuelle" en 1939, el primer museo pensado para existir exclusivamente en el ciberespacio es el Guggenheim Virtual Museum que Asymptote proyectó en 1999. En este proyecto, la idea de la Fundación no tenía que imitar la arquitectura del célebre Wright, sino más bien enseñar al mundo un espacio expositivo con su propia estética; un "superespacio" que los visitantes podían ir descubriendo en tiempo real, a través del interfaz VRML. 15. Rem Koolhaas insistió mucho sobre el tema de la "memoria" y con su uso de la palabra Preservation quiere romper el perverso - para él- frenesís de la conservación, y propone considerar tanto las excelencias reconocidas por códigos y reglas como lo ordinario y lo informal. Se recuerda el proyecto para Pekín del 2002, Bienal de Venecia de 2014 y, en el mismo año, el libro Preservation is overtaking us. Es interesante volver a proponerlo como método de intervención en aquellas operaciones de regeneración que se irán poco a poco difundiendo. Cf. Giani, E. (2012) - Rem Koolhaas: working with history, p. 1-3 y 8.

16. La acepción que atribuimos al dicho "Historia de las Mentalidades" (plural) se completa añadiendo el adjetivo colectivas. "La relación entre la Historia de las Mentalidades y la Historia de las Ideas es equivalente a la relación entre la Cultura Material y la Historia Económica”. Le Goff J. (1981) - La mentalitá: una storia ambigua. En: J. Le Goff y P. Nora, Fare Storia, pp. 243-244. Y P. Ariés (1999). Storia delle mentalitá. En: J. Le Goff y P. Nora, La Nuova Storia, p. 165ss. [E.Z.]

17. "La memoria colectiva y su forma científica, es decir la Historia, se aplican a dos tipos de material: los documentos y los monumentos. Lo que sobrevive no es el conjunto de lo que ha existido en el pasado, si no una elección dictada tanto por fuerzas que operan para enfocar la evolución temporal del mundo y de la humanidad, como para los que se 
dedican al estudio del pasado y de los tiempos pasados, es decir los historiadores. Dichos materiales de la memoria suelen tener dos formas de presentarse: los monumentos, herencias del pasado, y los documentos, elección de los historiadores". J. Le Goff (1978) - Documento/Monumento, pp. 38-43. [E.Z.]

18. "Hay un cuadro de Klee que se llama Angelus Novus. En ese cuadro se representa a un ángel que parece a punto de alejarse de algo a lo que mira fijamente. Los ojos se le ven desorbitados, tiene la boca abierta y, además, las alas desplegadas. Pues bien, este aspecto deberá tener el ángel de la historia. Él ha vuelto el rostro hacia el pasado. Donde ante nosotros aparece una cadena de datos (...). Pero, soplando desde el Paraíso, una tempestad se enreda en sus alas, y es tan fuerte que el ángel no puede cerrarlas. Esta tempestad lo empuja incontenible hacia el futuro, volviendo la espalda mientras el cúmulo de ruinas ante él crece hasta el cielo. Lo que llamamos progreso es justamente esta tempestad". Walter Bemjamin, Sobre el concepto de Historia, en Obras, Libro I, Vol. 2, p. 310 (2008).

19. Zeitgeist es una palabra compuesta de origen alemán: Seit (tiempo) y Geist (espíritu); el concepto define el alma o sentido de un periodo en la historia, como reflejo de las ideas y creencias del momento. El Zeitgeist se refiere a la ética y moral de una era y de un particular lugar como también al espíritu colectivo de un tiempo y un espacio cuyo reflejo es la propia cultura. Si la historia es resultados de las acciones de héroes y está escrita para los "ganadores", el Zeitgeist es influencia de unos pocos. El Zeitgeist es un reflejo vivo de nuestra identidad en transición y de todo lo que hacemos. En este artículo se usó la palabra Zeitgeist para traducir en alemán la expresión latina genius saeculi.

\section{Bibliografía}

Ariés, P. (1999). “Storia delle mentalità”. En: J. Le Goff, La Nuova Storia. Mondadori, Milano. Bemjamin, B. (2008). Obras. Libro I, Vol. 2, Madrid: Abada Editores.

Bucaille, R. y Pesez J. M. (1993). Cultura materiale. Ad vocem: Enciclopedia Einaudi, Vol. V. IV.

Burke, P. (1990). The French Historical Revolution: The Annales School 1929-89. Stanford, California: Stanford University Press.

Carnevale, G. (1989). "Il grottesco prossimo venturo". En: M. Canestrari, Architettura e forma urbana. Clean, Napoli.

Carnevale, G. (1993). “Deformazioni ai margini”. En Op.cit. n.76.

Carnevale, G. (1995). “Grottesco”, “A regola d'arte”. Ad vocem: L. Semerani, Dizionario illustrato delle voci più utili all'architetto moderno. Rizzoli, Bologna.

Carnevale, G. (1999). “Architettura grottesca, una non evitabile opportunità”. En: M. Montuori, Studi in onore di Giuseppe Samonà vol. 1. Roma: Officina ed.

Carnevale, G. (2006). A regola d'arte. Roma: Officina ed.

Carnevale, G. (2012). "Paradigmi Alieni". Actas del primero congreso internacional ReteVitruvio (Bari, 2-6 maio)

Carnevale, G. (2015). “Interstizi”. Actas del II congreso internacional sobre preservation, maintenance and rehabilitation of Historical Building and Structure REHAB (Porto, 21-24 julio). 
Carnevale, G. y Giani, E. (2014). Occasioni di ricerca. Il nuovo che arretra // Research opportunities. The retreat of the new. Milano: Maggioli ed.

Carnevale, G. y Giani, E. (2015). “The Urban Scene”. Actas del XXII congreso internacional: City as organism. New visions for urban life ISUF (Roma, 22-26 septiembre).

Di Stefano, R. (2003). La storia contemporanea nelle "Annales". FrancoAngeli, Milano.

Dostoevskij, F. (1869). L'Idiota.

Giani, E. (2012). “Rem Koolhaas: working with history”. En Iuav Giornale dell'Università n.120.

Giani, E. (2015a). “Travel in time. Is the informal a heritage to be preserved?" Actas del II congreso internacional sobre preservation, maintenance and rehabilitation of Historical Building and Structure REHAB (Porto, 21-24 julio).

Giani, E. (2015b). "Landscapes of repentance and of compensation". Actas del XII forum internacional de studios Le Vie dei mercanti. Heritage and Technology. Mind knowledge experience (Aversa-Capri, Italia 11-3 junio).

Giani, E. (2016). "For an operante archaeology of the present. Case studies: vernacular Venice and Berisso y Ensenada (La Plata, Argentina)". Actas del V congreso internacional sobre Heritage and Sustainable Development HERITAGE (Lisboa, 12-15 julio).

Le Goff, J.(1978). Documento/Monumento. Ad vocem: Enciclopedia Einaudi, Torino 1978, vol. V.

Le Goff , J. (1981). "Le mentalità: una storia ambigua”. In: J. Le Goff e P. Nora, Fare Storia pp. 243-244, Torino: Einaudi.

Ortega y Gasset J. (1993). La scelta in amore. Milano: Es edizioni.

Perec, G. (2011) . Tentativo di esaurimento di un luogo parigino. Roma: Voland ed.

Todorov, C. (2010). La bellezza salverà il mondo. Milano: Garzanti.

\begin{abstract}
Ptolemy created a place to gather the artistic and cultural production in a place: a building of worship, reserved for few selected people. Since the 1990s, the Museum becomes an international arena where Countries could confront and compare their cultural policies and management. The sphere of financial interests is closely linked to the production of intangible assets; public and private entities appeared to be rivals or allied, thus encouraging amateur collectors to expose their collection to the public. The knowledge fragmentation into multiple focused expertise lead to an increasingly specialized knowledge, changing the same representation of knowledge. Museums caught this as opportunity by gathering historical, social, and domestic segments that are increasingly fragmented too: yet, the development of this new concept of Museum is ongoing. To foreseen a possible scenario for the Idea of Museum, two questions arise: what to select, sort and preserve; and how to do it. What can be done to fix these slivers of the present mentality and of a shared aesthetic sensibility?

The issue involves deep ethical aspects, implies the questioning of functional, and economic mechanisms, calls for a responsibility towards the future, which is often the cause of dominant economic logic.
\end{abstract}


Keywords: Material culture - mentality - archaeology of the present.

Resumo: A beleza salvará o mundo. Poucas são as frases que foram usadas tanto quanto isso. A beleza está se manifestando de maneira cada vez mais impetuosa. Nos referimos à exigência de acessar a beleza como "alto conhecimento" em espaços culturais. Ptolomeu representou a produção artística e cultural, colocando-a em um local preciso: um edifício de culto, reservado aos poucos escolhidos. Desde a década de 1990, o Museu tornou-se uma área de confronto internacional para verificar as políticas culturais de vários países. Isso abre uma área de interesses financeiros, intimamente relacionada à produção de ativos intangíveis; o público e o privado aparecem em algumas ocasiões como antagonistas e em outros como aliados, aumentando o estabelecimento de museus particulares que abrem suas próprias coleções ao público. O desmembramento do conhecimento em competições cada vez mais especializadas modificou a representação do próprio conhecimento, e os museus o aproveitaram coletando memórias históricas, sociais e costumeiras, cada vez mais fragmentadas. Para definir uma possível idéia futura do Museu, dois aspectos precisam ser esclarecidos: primeiro o que será essencial para coletar, ordenar e transmitir e, segundo e mais importante, como fazê-lo. O que pode ser feito para amarrar fragmentos da atualidade, os de uma mentalidade contemporânea, os de uma sensibilidade estética compartilhada? Essa questão envolve questões éticas profundas, abrindo o debate sobre sistemas funcionais e econômicos que resultam em um chamado para assumir a responsabilidade pelo futuro e que, infelizmente, é frequentemente evitado devido à lógica econômica dominante.

Palavras chave: Beleza - Espaços Culturais - Paradigma Material - Arqueologia do Presente.

[Las traducciones de los abstracts fueron supervisadas por el autor de cada artículo] 